Pacific

Journal of

Mathematics

ALGEBRAIC $D$-GROUPS AND DIFFERENTIAL GALOIS THEORY

AnAnd Pillay

Volume $216 \quad$ No. 2

October 2004 


\title{
ALGEBRAIC D-GROUPS AND DIFFERENTIAL GALOIS THEORY
}

\author{
AnAND Pillay
}

\begin{abstract}
We discuss various relationships between the algebraic $D$ groups of Buium, 1992, and differential Galois theory. In the first part we give another exposition of our general differential Galois theory, which is somewhat more explicit than Pillay, 1998, and where generalized logarithmic derivatives on algebraic groups play a central role. In the second part we prove some results with a "constrained Galois cohomological flavor". For example, if $G$ and $H$ are connected algebraic $D$-groups over an algebraically closed differential field $F$, and $G$ and $H$ are isomorphic over some differential field extension of $F$, then they are isomorphic over some Picard-Vessiot extension of $F$. Suitable generalizations to isomorphisms of algebraic $D$-varieties are also given.
\end{abstract}

\section{Introduction}

We work throughout with (differential) fields of characteristic zero. In [8] the notion of a generalized differential Galois extension (or generalized strongly normal extension) of a differential field was introduced, generalizing Kolchin's theory of strongly normal extensions, which in turn generalized the Picard-Vessiot theory. The idea was to systematically replace algebraic groups over the constants by "finite-dimensional differential algebraic groups", to obtain new classes of extensions of differential fields with a good Galois theory. This idea (almost obvious from the model-theoretic point of view) was implicit in Poizat [11] who gave a model-theoretic treatment of the strongly normal theory. However the "correct" definition of a generalized differential Galois extension needed some additional fine-tuning. Nevertheless, our exposition of this general theory in [8] was overly model-theoretic, and possibly remained somewhat obscure to differential algebraists. We try to remedy this in the current paper by concentrating on the differential equations that have a good Galois theory, very much in the spirit of Section 7, Chapter IV of Kolchin's book [4]. The key notion is that of a generalized logarithmic derivative on an algebraic group $G$ over a differential field $K$ (a certain kind of differential rational map from $G$ to its Lie algebra). We 
will see that such a generalized logarithmic derivative is essentially equivalent to an algebraic $D$-group structure on $G$ (in the sense of Buium [3]). Our resulting exposition of the generalized differential Galois theory will be equivalent to that in [8] when the base field $K$ is algebraically closed. The general situation ( $K$ not necessarily algebraically closed) can be treated using analogues of the $V$-primitives from [4, IV.10], and we leave the details to others.

Let me now say a little more about the generalized logarithmic derivatives, and how they tie up with the Picard-Vessiot/strongly normal theory. Let us fix a differential field $K$, and assume for now that the field $C_{K}$ of constants of $K$ is algebraically closed. A linear differential equation over $K$, in vector form, is $\partial y=A y$, where $y$ is a $n \times 1$ column vector of unknowns and $A$ is an $n \times n$ matrix over $K$. Looking for a fundamental matrix of solutions, one is led to the equation on $\mathrm{GL}_{n}: \partial Y=A Y$, where $Y$ is a $n \times n$ matrix of unknowns ranging over $\mathrm{GL}_{n}$, which we can write as $\partial(Y) Y^{-1}=A$. Now the map $Y \rightarrow \partial(Y) Y^{-1}$ is the classical logarithmic derivative, a first-order differential crossed homomorphism from $\mathrm{GL}_{n}$ into its Lie algebra, which is surjective when viewed in a differentially closed overfield of $K$. A PicardVessiot extension of $K$ for the original equation is then a differential field extension $L=K(g)$, where $g \in \mathrm{GL}_{n}$ is a solution of $\partial(Y) Y^{-1}=A$, and $C_{L}=C_{K}$. Such an extension exists, and is unique up to $K$-isomorphism. The group of (differential) automorphisms of $L$ over $K$ has the structure of an algebraic subgroup of $\mathrm{GL}_{n}\left(C_{K}\right)$, and there is a Galois correspondence.

In place of $\mathrm{GL}_{n}$ one can consider an arbitrary algebraic group $G$ defined over $K$ (not necessarily linear and not necessarily defined over the constants of $K)$. By a generalized logarithmic derivative on $G$ we will mean a first-order differential rational crossed homomorphism $\mu$ from $G$ to $L(G)$, defined over $K$, such that $\mu$ is geometrically surjective (that is, surjective when viewed in a differentially closed overfield) and such that $\operatorname{Ker}(\mu)$, a finite-dimensional differential algebraic subgroup of $G$, is Zariski-dense in $G$. The analogue of a linear differential equation over $K$ will then be an equation

$$
\mu(x)=a,
$$

where $x$ ranges over $G$ and $a \in L(G)(K)$.

Under an additional technical condition on the data, analogous to the requirement that the field of constants of $K$ be algebraically closed, we can define the notion of a differential Galois extension $L$ of $K$ for the equation $(*)$, prove its existence and uniqueness, identify the Galois group, and obtain a Galois correspondence. In the case where $G$ is defined over the constant field $C_{K}$ and $\mu$ is the standard logarithmic derivative of Kolchin, we recover Kolchin's strongly normal extensions (see Theorem 6, Section 7, Chapter IV of $[4])$. 
For $G$ an algebraic group over the differential field $K$, an algebraic $D$ group structure on $G$ is precisely an extension of the derivation $\partial$ on $K$ to a derivation on the structure sheaf of $G$, respecting the group operation. Algebraic $D$-groups belong entirely to algebraic geometry, and Buium [3] points out that there is an equivalence of categories between the category of algebraic $D$-groups and the category of $\partial_{0}$-groups, finite-dimensional differential algebraic groups. The latter category belongs to Kolchin's differential algebraic geometry. On the other hand, there is essentially a one-to-one correspondence between algebraic $D$-group structures on $G$ and generalized logarithmic derivatives on $G$. So our general differential Galois theory is in a sense subsumed by the very concept of an algebraic $D$-group.

Details of the above will be given in Sections 2 and 3, including a "Tannakian" approach and an examination of different manifestations of the differential Galois group.

In Section 4 we will give another relation between algebraic $D$-groups and the Picard-Vessiot theory: if two algebraic $D$-groups over an algebraically closed differential field are isomorphic (as $D$-groups) over some differential field extension of $K$, then such an isomorphism can be found defined over a Picard-Vessiot extension of $K$. This uses Kolchin's differential Lie algebra, and strengthens the somewhat artificial results from [9]. We will also give some related results on isomorphisms between algebraic $D$-varieties, using differential jets (higher-dimensional versions of differential tangent spaces).

\section{Algebraic $D$-groups}

We will briefly describe the algebraic $D$-groups of Buium in a matter suitable for our purposes. We also introduce the generalized logarithmic derivative on an algebraic group induced by a given $D$-group structure. We refer to [6] for a discussion of related themes.

Let us fix an ordinary differential field $(K, \partial)$. For convenience we also give ourselves a differential field extension $(\mathcal{U}, \partial)$ of $(K, \partial)$ that is "universal" with respect to $(K, \partial)$. Namely, $\mathcal{U}$ has cardinality $\kappa>|K|$, and for any differential subfield $F<\mathcal{U}$ of cardinality $<\kappa$ and differential extension $L$ of $K$ of cardinality $\leq \kappa$ there is an embedding (as differential fields) of $L$ into $\mathcal{U}$ over $K . C_{K}$ denotes the field of constants of $K$, and $\mathcal{C}$ the field of constants of $\mathcal{U}$.

We begin by recalling the tangent bundle of an algebraic variety or group over $K$ (in which the derivation on $K$ plays no role).

Let $X$ be an algebraic variety over $K$ (maybe reducible). The tangent bundle $T(X)$ of $X$ is another algebraic variety over $K$, with a canonical surjective morphism $\pi$ (over $K$ ) to $X$, and is defined locally by equations: $\sum_{i} \partial P / \partial x_{i}\left(x_{1}, \ldots, x_{n}\right) v_{i}=0$ for $P$ polynomials over $K$ generating the ideal of $X$ over $K$. If $X=G$ is an algebraic group over $K$, then $T(G)$ has the 
structure of an algebraic group over $K$ such that the canonical projection to $G$ is an (algebraic) group homomorphism. The group operation on $T(G)$ is obtained by differentiating the group operation of $G$. That is, if $f(-,-)$ is the group operation on $G$ and $(g, u)$ and $(h, v)$ are in $T(G)$ then the product $(g, u) \cdot(h, v)$ equals $\left(g \cdot h, d f_{g, h}(u, v)\right)$. Note that if $\lambda^{g}, \rho^{g}$ denote left and right multiplication by $g$ in $G$, then we have:

$$
\text { In } T(G), \quad(g, u) \cdot(h, v)=\left(g \cdot h, d\left(\lambda^{g}\right)_{h}(v)+d\left(\rho^{h}\right)_{g}(u)\right) .
$$

We will denote $T(G)_{e}$, the tangent space of $G$ at the identity, by $L(G)$ (for the Lie algebra of $G$ ). Then $L(G)$, with its usual vector space group structure, is a normal subgroup of $T(G)$, and we have the exact sequence $0 \rightarrow L(G) \rightarrow T(G) \rightarrow G \rightarrow\{e\}$ of algebraic groups (over $K$ ). We denote by $i: L(G) \rightarrow T(G)$ the natural inclusion, and by $\pi: T(G) \rightarrow G$ the canonical surjection above.

Note that $G$ acts on $L(G)$ (denoted $(g, a) \rightarrow a^{g}$ ) by the adjoint map (differentiating conjugation by $g \in G$ at the identity). And this "coincides" with the action of $T(G)$ on the normal subgroup $L(G)$ by conjugation: for $a \in L(G)$ and $g \in G, a^{g}=x a x^{-1}$ for any $x \in T(G)$ such that $\pi(x)=g$.

For $a \in L(G)$, we let $l_{a}$ and $r_{a}$ denote the left and right invariant vector fields on $G$ determined by $a$. Namely for $g \in G, l_{a}(g)=d\left(\lambda^{g}\right)_{e}(a)$ and $r_{a}(g)=d\left(\rho^{g}\right)_{e}(a)$.

A $K$-rational splitting of $T(G)$ as a semidirect product of $G$ and $L(G)$ is given by either of the equivalent pieces of data:

(a) a $K$-rational homomorphic section $s: G \rightarrow T(G)$ (that is $\pi \circ s=\mathrm{id}$ );

(b) a $K$-rational crossed homomorphism $h$ from $T(G)$ onto $L(G)$ such that $h \circ i=\mathrm{id}$. (By definition $\alpha: T(G) \rightarrow L(G)$ is a crossed homomorphism if $\alpha(x y)=\alpha(x)+x \alpha(y) x^{-1}$.)

Note that the set of these $K$-rational splittings has the structure of a commutative group. For example, using the data in (a), if $s_{1}, s_{2}$ are $K$ rational homomorphic sections of the tangent bundle, and $s_{i}(g)=\left(g, u_{i}\right)$ for $i=1,2$ then $\left(s_{1}+s_{2}\right)(g)=\left(g, u_{1},+u_{2}\right)$. The identity element is just the 0 -section $s_{0}(g)=(g, 0)$, which is a $K$-rational homomorphic section. Let $P_{K}(G)$ denote this commutative group of $K$-rational splittings of $T(G)$. We denote the crossed homomorphism from $T(G)$ onto $L(G)$ corresponding to the identity of $P_{K}(G)$ by $h_{0}: T(G) \rightarrow L(G)$, and the crossed homomorphism corresponding to the homomorphic section $s$ by $h_{s}$.

\section{Remark 2.1.}

(i) $h_{0}(g, u)=d\left(\rho^{g^{-1}}\right)_{g}(u)$.

(ii) More generally, if $s$ is a $K$-rational homomorphic section of $T(G) \rightarrow G$, then $h_{s}(g, u)=d\left(\rho^{g^{-1}}\right)_{g}(u-s(g))$.

Proof. This follows directly from formula $(*)$ for multiplication in $T(G)$. 
Let us now bring in the differential structure. Assume for now that the algebraic variety $X$ is defined over $C_{K}$ the field of constants of $K$. Then it is easy to see that if $a \in X(\mathcal{U})$ then working in local coordinates with respect to a given covering of $X$ by affine varieties over $C_{K},(a, \partial(a)) \in T(X)$. If in addition $X=G$ is an algebraic group defined over $C_{K}$, then the map $\nabla: G(\mathcal{U}) \rightarrow T(G)(\mathcal{U})$ taking $g$ to $(g, \partial(g))$ is a group embedding. Let $l D: G(\mathcal{U}) \rightarrow L(G)(\mathcal{U})$ be defined by $l D(g)=h_{0}(g, \partial(g))$. Then $l D$ is a "differential rational" crossed homomorphism defined over $K$, which is precisely Kolchin's logarithmic derivative. The map $l D$ depends on $h_{0}$, and clearly any other $h \in P_{K}(G)$ gives rise to another differential rational crossed homomorphism (over $K$ ) from $G(\mathcal{U})$ onto $L(G)(\mathcal{U})$.

Remark 2.2. Suppose $G$ is defined over $C_{K}$. Then:

(i) The (standard) logarithmic derivative $l D: G(\mathcal{U}) \rightarrow L(G)(\mathcal{U})$ is given by $l D(g)=d\left(\rho^{g^{-1}}\right)_{g}(\partial(g))$.

(ii) $l D$ is surjective.

(iii) $\operatorname{Ker}(l D)$ is precisely $G(\mathcal{C})$.

Proof. (i) follows from Remark 2.1.

(ii) Let $a \in L(G)(\mathcal{U})$. Then $a$ determines the right invariant vector field $r_{a}: G(\mathcal{U}) \rightarrow T(G)(\mathcal{U})$. As $\mathcal{U}$ is differentially closed, the main result of $[7]$ gives $g \in G(\mathcal{U})$ such that $\partial(g)=r_{a}(g)$, hence (by (i)), $l D(g)=a$.

(iii) is obvious from (i).

Let us now work in a more general context, dropping our assumption that the variety $X$ is defined over $C_{K}$. Then for $a \in X(\mathcal{U}),(a, \partial(a))$ may no longer be a point of $T(X)$ but rather a point of another bundle $\tau(X)$ over $X$, which we now describe. For $P\left(x_{1}, \ldots, x_{n}\right)$ a polynomial over $K$, let $P^{\partial}$ denote the polynomial obtained from $P$ by applying $\partial$ to its coefficients. (So $P^{\partial}=0$ if $P$ is over $C_{K}$.) Then $\tau(X)$ is defined locally by equations:

$$
\sum_{i} \partial P / \partial x_{i}\left(x_{1}, \ldots, x_{n}\right) v_{i}+P^{\partial}\left(x_{1}, \ldots, x_{n}\right)=0,
$$

where $P$ again ranges over polynomials in the ideal of $X$ over $K$. (That is, these affine pieces fit together to give an algebraic variety $\tau(X)$ over $K$, together with a canonical projection from $\tau(X)$ to $X$.) It is immediate that $(a, \partial a) \in \tau(X)$ for $a \in X(\mathcal{U})$. It is also immediate that for each $a \in X$, $\tau(X)_{a}$ is a principal homogeneous space for the tangent space $T(X)_{a}$, where the action is addition (with respect to local coordinates above). Moreover this happens uniformly, making $\tau(X)$ a torsor for the tangent bundle $T(X)$. In any case, if $X$ is defined over $C_{K}$, then $\tau(X)$ coincides with $T(X)$. By an algebraic $D$-variety over $K$ we mean a pair $(X, s)$ such that $X$ is an algebraic variety over $K$ and $s: X \rightarrow \tau(X)$ is a regular section defined over $K$. 
Now assume again that $X=G$ is an algebraic group over $K$. Then $\tau(G)$ has the structure of an algebraic group over $K$ such that the canonical projection $\tau(G) \rightarrow G$ is a homomorphism. In local coordinates, assuming that multiplication in $G$ is given by the sequence of polynomials $f=\left(f_{i}\left(x_{1}, \ldots, x_{n}, y_{1}, \ldots, y_{n}\right)\right)_{i=1, \ldots, n}$ over $K$, then for $(g, u),(h, v) \in \tau(G)$, the product of $(g, u)$ and $(h, v)$ in $\tau(G)$ is given by

$$
\left(g \cdot h, d f_{(g, h)}(u, v)+\left(f_{1}^{\partial}(g, h), \ldots, f_{n}^{\partial}(g, h)\right)\right) .
$$

We again have the map $\nabla: G(\mathcal{U}) \rightarrow \tau(G)(\mathcal{U})$, given (in local coordinates) by $\nabla(g)=(g, \partial(g))$ and this is a group embedding.

So we have two $K$-algebraic groups $T(G)$ and $\tau(G)$. Although these need not be isomorphic as algebraic groups, they are "differential rationally" isomorphic. The following is left to the reader.

Lemma 2.3. The map that takes $(g, u)$ to $(g, \partial(g)-u)$ is a (differential rational) isomorphism of groups between $\tau(G)(\mathcal{U})$ and $T(G)(\mathcal{U})$. Although not necessarily rational, it is rational when restricted to the fibers over $G$. In particular, the above map defines a $K$-rational isomorphism between the vector groups $\tau(G)_{e}$ and $T(G)_{e}=L(G)$.

Note that we have again an exact sequence

$$
0 \rightarrow \tau(G)_{e} \rightarrow \tau(G) \rightarrow G \rightarrow e
$$

of algebraic groups over $K$, which by virtue of the (canonical) isomorphism between $\tau(G)_{e}$ and $L(G)$ given by Lemma 2.3 can be rewritten as:

$$
0 \rightarrow L(G) \rightarrow \tau(G) \rightarrow G \rightarrow e .
$$

Let us again write $i$ for the (canonical) injection of $L(G)$ in $\tau(G)$, and $\pi$ for the canonical surjection $\tau(G) \rightarrow G$. So if $G$ is defined over $C_{K}$ this agrees with our earlier notation: $i: L(G) \rightarrow T(G)$ and $\pi: T(G) \rightarrow G$.

We can consider splittings (as algebraic groups over $K$ ) of $\tau(G)$ as a semidirect product of $G$ and $L(G)$. Again each such splitting is determined either by a $K$-rational homomorphic section $s: G \rightarrow \tau(G)$, or a $K$-rational crossed homomorphism $h: \tau(G) \rightarrow L(G)$ such that $h \circ i=$ id on $L(G)$. We will write $h_{s}$ for the crossed homomorphism corresponding to the homomorphic section $s$, and give explicit formulas below.

In any case, we can now define an algebraic $D$-group.

Definition 2.4. Let $G$ an algebraic group over $K$. Then an algebraic $D$ group structure on $G$ over $K$ is precisely a $K$-rational homomorphic section $s: G \rightarrow \tau(G)$. We write the corresponding algebraic $D$-group as $(G, s)$.

Given an algebraic $D$-group $(G, s)$ we obtain a generalized logarithmic derivative that we call $l D_{s}$, a crossed homomorphism (in the obvious sense) from $G(\mathcal{U})$ to $L(G)(\mathcal{U}): l D_{s}=h_{s} \circ \nabla$. Here is the analogue to Remarks 2.1 and 2.2 . 
Remark 2.5. Let $(G, s)$ be an algebraic D-group over $K$.

(i) For $(g, u) \in \tau(G), h_{s}(g, u)=d\left(\rho^{g^{-1}}\right)_{g}(u-s(g))$.

(ii) For $g \in G(\mathcal{U}), l D_{s}(g)=d\left(\rho^{g^{-1}}\right)_{g}(\partial(g)-s(g)) \in L(G)(\mathcal{U})$.

(iii) $l D_{s}: G(\mathcal{U}) \rightarrow L(G)(\mathcal{U})$ is surjective.

(iv) $\operatorname{Ker}\left(l D_{s}\right)$ is precisely $\{g \in G(\mathcal{U}): \partial(g)=s(g)\}$, and is a Zariski-dense subgroup of $G(\mathcal{U})$.

Proof. (i) follows from the formula for multiplication in $\tau(G)$, and (ii) is an immediate consequence of (i).

(iii) Let $a \in L(G)(\mathcal{U})$. Again we obtain the right invariant vector field $r_{a}: G(\mathcal{U}) \rightarrow T(G)(\mathcal{U})$. Then $r_{a}+s: G(\mathcal{U}) \rightarrow \tau(G)(\mathcal{U})$ is also a rational section of $\tau(G) \rightarrow G$. By [7] there is $g \in G(\mathcal{U})$ such that $\partial(g)=r_{a}(g)+s(g)$, hence $l D_{s}(g)=a$ by (i).

(iv) $\operatorname{Ker}\left(l D_{s}\right)$ is a subgroup of $G(\mathcal{U})$ as $l D_{s}$ is a crossed homomorphism. As $d\left(\rho^{g^{-1}}\right)_{g}$ is an isomorphism between $T(G)_{g}$ and $T(G)_{e}$, we see that $\operatorname{Ker}\left(l D_{s}\right)$ is as described in (iii). By [7] for any proper subvariety $X$ of $G(\mathcal{U})$ there is $g \in G(\mathcal{U})$ such that $\partial(g)=s(g)$. Hence by (i) $\operatorname{Ker}\left(l D_{s}\right)$ is Zariski dense in $G$.

In the context of Remark 2.5, we denote $\operatorname{Ker}\left(l D_{s}\right)$ by $(G, s)^{\sharp}$. This is a finite-dimensional differential algebraic group, or $\partial_{0}$-group in the sense of [3], and is an object belonging to Kolchin's differential algebraic geometry. Just for the record, here are some key properties: $(G, s)^{\sharp}(\mathcal{U})$ is Zariski-dense in $G$, the $\partial_{0}$-subvarieties of $(G, s)^{\sharp}$ are precisely of the form $X \cap(G, s)^{\sharp}$ for $X$ a $D$-subvariety of $(G, s)$, and for any other algebraic $D$-group $(H, t)$ the $\partial_{0}$-homomorphisms between $(G, s)^{\sharp}$ and $(H, t)^{\sharp}$ are precisely those induced by algebraic $D$-group homomorphisms between $(G, s)$ and $(H, t)$.

In any case, we have seen that an algebraic $D$-group structure $(G, s)$ on an algebraic group $G$ over $K$ determines a generalized logarithmic derivative $l D_{s}$ on $G$. We point out now, just for completeness, that conversely any suitable differential rational crossed homomorphism map (over $K$ ) from $G(\mathcal{U})$ to $L(G)(\mathcal{U})$ determines an algebraic $D$-group structure on $G$.

Some notation: Let $X$ and $Y$ be algebraic varieties defined over $K$. By a first-order differential rational map $h: X(\mathcal{U}) \rightarrow Y(\mathcal{U})$, defined over $K$, we mean a map $h$ from $X(\mathcal{U})$ to $Y(\mathcal{U})$ such that for, for each $x \in X(\mathcal{U})$, $h(x) \in K(x, \partial(x))$.

Lemma 2.6. Suppose that $G$ is a connected algebraic group over $K$. Let $l: G(\mathcal{U}) \rightarrow L(G)(\mathcal{U})$ be a first-order differential rational crossed homomorphism, defined over $K$, which is surjective, and is such that $\operatorname{Ker}(l)$ is $Z$ ariski-dense in $G(\mathcal{U})$. Then, there are a unique $K$-rational automorphism $\sigma$ of $L(G)$, and a unique algebraic D-group structure $(G, s)$ on $G$ (defined over $K)$, such that $l=\sigma \circ l D_{s}$. 
Proof. By [7], for example, $\nabla(G(\mathcal{U}))$ is Zariski-dense in $\tau(G)(\mathcal{U})$. Define $l_{1}$ on $\nabla(G(\mathcal{U}))$ by $l_{1}(x, \partial(x))=l(x)$. So by Zariski-denseness, and the properties of $l, l_{1}$ extends uniquely to a $K$-rational surjective crossed homomorphism $f$ from $\tau(G)$ to $L(G)$.

By the Zariski-denseness of $\operatorname{Ker}(l)$ in $G$ and the definition of $f$, we have $\pi(\operatorname{Ker}(f))=G$. On the other hand, $\operatorname{dim}(\tau(G))=2 \operatorname{dim}(G)$ and $\operatorname{dim}(G)=$ $\operatorname{dim}(L(G))$. Hence $\operatorname{dim}(\operatorname{Ker}(f))=\operatorname{dim}(G)$. Now $\pi \mid \operatorname{Ker}(f): \operatorname{Ker}(f) \rightarrow G$ is a group homomorphism so it has finite kernel. But this finite kernel is a subgroup of the vector group $\tau(G)_{e}$ so has to be trivial. It follows that $f \mid \tau(G)_{e}$ is an isomorphism (over $K$ ) with $L(G)$. So there is a unique $K$ automorphism $\sigma$ of $L(G)$ such that $(\sigma \circ f) \circ i$ is the identity on $L(G)$. Put $f_{1}=\sigma \circ f$. Then $f_{1}$ gives an algebraic $D$-group structure $(G, s)$ on $G$ defined over $K$. For $g \in G(\mathcal{U}), \sigma \circ l(g)=f_{1}(g, \partial(g))=l D_{s}(g)$.

There is a natural notion of a $D$-morphism between algebraic $D$-varieties $(X, s)$ and $(Y, t)$. First note that $\tau(-)$ is a functor, so if $f: X \rightarrow Y$ is a morphism between the algebraic varieties $X$ and $Y$ with everything defined over $K$ then $\tau(f)$ is a morphism, over $K$, between $\tau(X)$ and $\tau(Y)$, again defined over $f$. (If $X, Y, f$ are defined over the constants, then $\tau(f)$ is just the differential of $f$.) In any case, a morphism between algebraic $D$-varieties $X$ and $Y$ is by definition a morphism $f$ of algebraic varieties, such that $t \circ f=\tau(f) \circ s$.

In particular we extract the notion of an algebraic $D$-subvariety of $(X, s)$ : so an algebraic subvariety $Y$ of $X$ will be the underlying variety of an algebraic $D$-subvariety of $(X, s)$ if $s \mid Y$ maps $Y$ to $\tau(Y)$.

A homomorphism of algebraic $D$-groups is a $D$-morphism that is also a homomorphism of algebraic groups.

We call an algebraic $D$-group $(G, s)$ isotrivial if it is isomorphic over $\mathcal{U}$ to a trivial algebraic $D$-group $\left(H, s_{0}\right)$, where $H$ is defined over $\mathcal{C}$ and $s_{0}$ is the 0 -section of $T(G)$.

The interest of the category of algebraic $D$-groups is that there exist nonisotrivial algebraic $D$-groups. If $A$ is an abelian variety over $\mathcal{U}$ and $p: G \rightarrow A$ is the universal extension of $A$ by a vector group then $G$ has a $D$-group structure (defined over the field over which $A$ is defined). Moreover any such $D$-group structure on $G$ is nonisotrivial if $A$ is not isomorphic (as an algebraic group) to an abelian variety defined over $\mathcal{C}$. Given such a $D$ group structure $(G, s)$ on $G, p\left((G, s)^{\sharp}\right)<A$ is precisely the Manin kernel of $A$. This example is worked out in detail in $[6]$.

Let us repeat from [9] the discussion of a nonisotrivial algebraic $D$-group structure on the commutative algebraic group $G_{m} \times G_{a}$. So let $G=G_{m} \times G_{a}$. Then $T(G)=\tau(G)$ can be identified with $\{(x, y, u, v): x \neq 0\}$ with group structure $\left(x_{1}, y_{1}, u_{1}, v_{1}\right) \cdot\left(x_{2}, y_{2}, u_{2}, v_{2}\right)=\left(x_{1} x_{2}, y_{1}+y_{2}, u_{1} x_{2}+u_{2} x_{1}, v_{1}+v_{2}\right)$. 
Let $s: G \rightarrow T(G)$ be the homomorphic section $s(x, y)=(x, y, x y, 0)$. Then $(G, s)$ is an algebraic $D$-group known to be nonisotrivial.

Note that if $g=(x, y) \in G$, then the differential of multiplication by $g^{-1}$ at $g$ takes $(u, v) \in T(G)_{g}$ to $(u / x, v) \in L(G)$. Hence by 2.5 , the generalized logarithmic derivative $l D_{s}$ corresponding to $s$ is: $l D_{s}(x, y)=$ $((\partial(x)-x y) / x, \partial(y))=(\partial(x) / x-y, \partial(y))$.

In particular $(G, s)^{\sharp}=\operatorname{Ker}\left(l D_{s}\right)=\{(x, y) \in G: \partial(x) / x=y, \partial(y)=0\}$, which can be identified with the subgroup of $G_{m}$ defined by the second-order equation $\partial(\partial(x) / x)=0$.

\section{Differential Galois theory}

The conventions of the previous section are in force. In particular $(K, \partial)$ is a differential field of characteristic 0 , and we may refer also to the universal differential field extension $(\mathcal{U}, \partial)$ of $K$.

By a logarithmic differential equation over a differential field $(K, \partial)$ we mean something of the form

$$
l D_{s}(x)=a,
$$

where $(G, s)$ is an algebraic $D$-group defined over $K$ and $a \in L(G)(K)$. (So the indeterminate $x$ ranges over $G$.) As remarked in the introduction, a special case is the equation $\partial(X)=A X$, where $X$ ranges over $\mathrm{GL}_{n}$ and $A$ is an $n \times n$ matrix over $K$.

In order to give the right notion of a differential Galois extension of $K$ for $(*)$, we need to place a further restriction on $K, G$ and $s$.

Definition 3.1. Suppose $(G, s)$ is an algebraic $D$-group over $K$. We say that $(G, s)$ is $K$-large, if for every (maybe reducible) algebraic $D$-subvariety $X$ of $G$, which is defined over $K, X(K) \cap(G, s)^{\sharp}$ is Zariski-dense in $X$.

\section{Remark 3.2.}

(i) The intuitive meaning of $(G, s)$ being $K$-large is that $(G, s)^{\sharp}$ has enough points with coordinates in $K$.

(ii) Suppose that $G$ is defined over $C_{K}$ and that $s=s_{0}$, the 0 -section of $T(G)$. Then, if $C_{K}$ is algebraically closed, $(G, s)$ is $K$-large.

In the next remark, we refer to differential closures of $K$. A differential closure of $K$ is a differential field extension of $K$ that embeds over $K$ into any differentially closed field containing $K$. The differential closure of $K$ is unique up to $K$-isomorphism, and is written as $\hat{K}$. Kolchin calls $\hat{K}$ the constrained closure of $K$.

Remark 3.3. $(G, s)$ is $K$-large if and only $(G, s)^{\sharp}(K)=(G, s)^{\sharp}(\hat{K})$ for some (any) differential closure $\hat{K}$ of $K$. 
Proof. Assume first that $(G, s)^{\sharp}(K)=(G, s)^{\sharp}(\hat{K})$. Let $X$ be a $D$-subvariety of $(G, s)$ defined over $K$. The irreducible components $X_{1}, \ldots, X_{r}$ of $X$ are defined over $\bar{K}$ so also $\hat{K}$ and are also $D$-subvarieties of $(G, s)$. By [7] for example for any nonempty Zariski open subset of any $X_{i}$ defined over $\hat{K}$, there is $a \in U\left(\hat{K}\right.$ such that $\partial(a)=s(a)$. By our assumptions $a \in(G, s)^{\sharp}(K)$. So $X \cap(G, s)^{\sharp}(K)$ is Zariski-dense in $X$.

Conversely, suppose $(G, s)$ is $K$-large. Let $a \in(G, s)^{\sharp}(\hat{K})$, and suppose for a contradiction that $a \notin G(K)$. Now $a$ is constrained over $K$ in the sense of Section 10, Chapter III of [4]. This means that there is some differential polynomial $P(x)$ over $K$ such that $P(a) \neq 0$, and whenever $b$ is a differential specialization of $a$ over $K$ and $P(b) \neq 0$ then $b$ is a "generic" specialization of $a$ over $K$ (in model-theoretic language $t p(b / K)=t p(a / K)$ ). Let $X$ be the irreducible $K$-subvariety of $G$ whose generic point is $a$. Then (as $\partial(a)=$ $s(a)), X$ is a $D$-subvariety of $(G, s)$. Moreover the differential specializations of $a$ over $K$ are precisely those $b$ such that $b \in X$ and $\partial(b)=s(b)$. Now subject to the conditions " $x \in X$ and $\partial(x)=s(x)$ ", the condition $P(x) \neq 0$ is clearly equivalent to $x \notin Y$ for $Y$ some proper subvariety of $X$ defined over $K$. By our assumptions there is $b \in G(K)$ such that $b \in X \backslash Y$ and $\partial(b)=s(b)$. This is a contradiction.

We call a differential ring $(R, \partial)$ simple if it has no proper nontrivial differential ideals. We refer to [12] for a discussion of simple differential rings.

Lemma 3.4. Suppose that $R$ is a simple differential ring over $K$ that is finitely generated over $K$. Then $R$ embeds over $K$ into some differential closure of $K$.

Proof. As $R$ has no zero-divisors, $R$ embeds in $\mathcal{U}$ over $K$. Let $R=K[a]_{\partial}$ be differentially generated over $K$ by the finite tuple $a$. Suppose that $\pi(a)=b$ is a differential specialization over $K$. Then $\pi$ extends to a surjective ring homomorphism $\pi: R \rightarrow K[b]_{\partial}$. The kernel is a differential ideal, so must be trivial. Thus $b$ is a generic specialization of $a$ over $K$. It follows that $a$ is constrained over $K$ so lives in some differential closure of $K$, as does $R$.

We can now give the main definition.

Definition 3.5. Let $(G, s)$ be a $K$-large algebraic $D$-group defined over $K$, and $l D_{s}(x)=a$ be a logarithmic differential equation over $K$ for $(G, s)$. By a differential Galois extension of $K$ for the equation $l D_{s}=a$ we mean a differential field extension $L$ of $K$ of the form $K(\alpha)$ for some solution $\alpha$ of the equation such that $K[\alpha]$, the (differential) ring generated by $K$ and the coordinates of $\alpha$, is a simple differential ring.

Lemma 3.6 (Existence and uniqueness of differential Galois extensions). If $(G, s)$ is a $K$-large algebraic D-group defined over $K$, and $a \in L(G)(K)$, 
then there exists a differential Galois extension $L$ of $K$ for the equation $l D_{s}(x)=a$. Moreover, any two such extensions are isomorphic over $K$ as differential fields.

Proof. By Remark 2.5 (iii) there is a solution $\beta \in G(\mathcal{U})$ of $l D_{s}(x)=a$. Let $\alpha$ be a maximal differential specialization of $\beta$ over $K$. Then $K[\alpha]$ is a simple differential ring and $\alpha$ is also a solution of $l D_{s}=a$, so we get existence. Let $L=K(\alpha)$.

Suppose $L_{1}$ is another differential Galois extension of $K$ for the equation, generated by the solution $\gamma$ say. By Lemma 3.4 we may assume that both $L$ and $L_{1}$ are contained in some differential closure $\hat{K}$ of $K$. By Remark 3.3, $(G, s)^{\sharp}(\hat{K})=(G, s)^{\sharp}(K)$. As both $\alpha$ and $\gamma$ are solutions of $l D_{s}(x)=a$, it follows that $\alpha^{-1} \cdot \gamma \in(G, s)^{\sharp}(\hat{K})=(G, s)^{\sharp}(K)$. Thus clearly $L=L_{1}$.

Here are some alternative characterizations of differential Galois extensions:

Lemma 3.7. Let $(G, s)$ be a $K$-large algebraic D-group over $K$, and $L=$ $K(\alpha)$ a differential field extension of $K$, where $\alpha$ is a solution of $l D_{s}(x)=a$ (with $a \in L(G)(K)$ ). Then the following are equivalent:

(i) $L$ and $\alpha$ satisfy Definition 3.5.

(ii) $L$ is contained in some differential closure of $K$.

(iii) $(G, s)^{\sharp}(K)=(G, s)^{\sharp}(L)$ and $(G, s)$ is L-large.

Proof. (i) implies (ii) is given by Lemmas 3.4 and 3.6 and (ii) implies (iii) follows from Remark 3.3.

(iii) implies (i). Assume $L$ satisfies (iii). Then using Remark 3.3, we have that $(G, s)^{\sharp}(\hat{L})=(G, s)^{\sharp}(K)$. Now $\hat{K}$ embeds in $\hat{L}$ over $K$, hence by Lemmas 3.4 and 3.6, there is a solution $\beta \in G(\hat{L})$ of $l D_{s}(x)=a$, such that $K[\beta]$ is a simple differential ring. Now $\alpha=\beta \cdot g$ for some $g \in(G, s)^{\sharp}(K)$, so clearly $K[\alpha]$ is also a simple differential ring.

Condition (iii) is the analogue of "no new constants" in the strongly normal case.

Remark 3.8. Suppose $K$ is algebraically closed. Then the differential Galois extensions of $K$ in the sense of Definition 3.5 coincide with the generalized strongly normal extensions of $K$ in the sense of [8]. In particular, $L$ is a strongly normal extension of $K$ (in the sense of Kolchin) just if $L$ is a differential Galois extension of $K$ for an equation $l D_{s}(x)=a$, on an algebraic D-group $(G, s)$, where $G$ is defined over $C_{K}$ and $s=s_{0}$.

Proof. This follows from Proposition 3.4 of [8].

We now point out that given $L$ as above, $\operatorname{Aut}_{\partial}(L / K)$ has the structure of a differential algebraic group (over $K$ ) in two different ways. One corresponds to the usual differential Galois group in the linear case, and is simply of the 
form $(H, s)^{\sharp}(K)$, where $H$ is an algebraic $D$-subgroup of $(G, s)$. The other, corresponding to the "intrinsic" Galois group introduced by Katz, is of the form $\left(H_{1}, s_{1}\right)^{\sharp}(L)$ for $s_{1}$ another algebraic $D$-group structure on $G$ (defined over $K)$, and $H_{1}$ a $D$-subgroup of $\left(G, s_{1}\right)$ defined over $K$. The algebraic $D$-groups $(H, s)$ and $\left(H_{1}, s_{1}\right)$ will be isomorphic, but not necessarily over $K$, unless they are commutative. (But of course if $K$ is algebraically closed, $H$ and $H_{1}$ will be isomorphic over $K$ as algebraic groups.)

So fix $L=K(\alpha)$ as in Definition 3.5. By 3.7 we have $L<\hat{K}$ for some fixed copy of the differential closure of $K$. $\operatorname{Aut}_{\partial}(L / K)$ denotes the group of differential field automorphisms of $L$ over $K$. Note that for any $\sigma \in$ $\operatorname{Aut}_{\partial}(L / K), \sigma(\alpha)$ is also a solution of $l D_{s}=a$, hence $\sigma(\alpha)=b \cdot c(\sigma)$ for a unique $c(\sigma) \in(G, s)^{\sharp}(L)=(G, s)^{\sharp}(K)\left(=(G, s)^{\sharp}(\hat{K})\right)$.

Lemma 3.9. The map c above is a group isomorphism between $\operatorname{Aut}_{\partial}(L / K)$ and $(H, s)^{\sharp}(K)$ for some algebraic $D$-subgroup $H$ of $G$ defined over $K$.

Proof. As an automorphism $\sigma$ of $L$ over $K$ is determined by its action on $\alpha$, clearly the map is a group isomorphism with its image. So all that we need is that the image is of the required form. The model-theoretic proof of this goes through showing that the image is a definable subgroup of $(G, s)^{\sharp}(\hat{K})$, and then using quantifier-elimination for differentially closed fields. We will give an algebraic proof, after first discussing the second incarnation of the Galois group.

First, the equation $l D_{s}(x)=a$ equips $G$ with another structure of an algebraic $D$-variety (but not in general an algebraic $D$-group). Let $r_{a}$ be the right invariant vector field on $G$ determined by $a$ on $G$. So $s+r_{a}$ is a $K$-rational section of $\tau(G) \rightarrow G$, which we denote by $s^{\prime}$. Note that the equation $l D_{s}(x)=a$ on $G$ is equivalent to the equation $\partial(x)=s^{\prime}(x)$.

Now $G$ acts on itself by left translation. Let $S<G$ be the intersection of the stabilizers of the algebraic $D$-subvarieties of the algebraic $D$-variety $\left(G, s^{\prime}\right)$ that are defined over $K$ : namely (working in some algebraically closed field containing $K), S=\{g \in G: g \cdot X=X$ for all $D$-subvarieties $X$ of $\left(G, s^{\prime}\right)$ defined over $\left.K\right\}$. $S$ is an algebraic subgroup of $G$ defined over $K . S$ is precisely the analogue in our context of the intrinsic differential Galois group introduced by Katz in the Picard-Vessiot case and discussed by Bertrand in $[\mathbf{1}]$.

In fact $G$ can be naturally equipped with another structure of an algebraic $D$-group, $\left(G, s_{1}\right)$. Define the section $s_{1}: G \rightarrow \tau(G)$ by

$$
s_{1}(g)=s(g)+r_{a}(g)-l_{a}(g) .
$$

Lemma 3.10. $\left(G, s_{1}\right)$ is an algebraic D-group, and the action of $G$ on itself by left multiplication is an action of the algebraic $D$-group $\left(G, s_{1}\right)$ on the algebraic D-variety $\left(G, s^{\prime}\right)$. Moreover $S$ is an algebraic D-subgroup of $\left(G, s_{1}\right)$. 
Proof. An easy computation. Note that it follows that $\left(G, s_{1}\right)^{\sharp}(\mathcal{U})$ acts on $\left(G, s^{\prime}\right)^{\sharp}(\mathcal{U})$.

Let $Z$ be the set of solutions of $l D_{s}(x)=a$ in $G(L)$. Note that $Z$ is precisely $\left(G, s^{\prime}\right)^{\sharp}(L)$. In any case, $Z$ is a principal homogeneous space for $(G, s)^{\sharp}(K)$ (acting on the right). As $(G, s)^{\sharp}(K)=(G, s)^{\sharp}(\hat{K})$, and $L<\hat{K}$, $X$ is also the solution set of $l D_{s}(x)=a$ in $G(\hat{K})$. Clearly Aut ${ }_{\partial}(L / K)$ acts on $Z$ and any $\sigma \in \operatorname{Aut}_{\partial}(L / K)$ is determined by its action on $Z$. In fact, as $L=K(\beta)$ for any $\beta \in Z, \sigma \in \operatorname{Aut}_{\partial}(L / K)$ is determined by any pair $(\beta, \sigma(\beta))$ for $\beta \in Z$.

Lemma 3.11. The action of $\operatorname{Aut}_{\partial}(L / K)$ on $Z$ is isomorphic to the action (by left multiplication) of $\left(S, s_{1}\right)^{\sharp}(L)$ on $Z$ : The isomorphism d say takes $\sigma$ to $\sigma(\beta) \cdot \beta^{-1}$ for some (any) $\beta \in Z$.

Proof. We know that $\left(S, s_{1}\right)^{\sharp}$ acts on $Z$ by left multiplication (by Lemma 3.10). Suppose $\sigma \in \operatorname{Aut}_{\partial}(L / K)$. Let $d(\sigma) \in G(L)$ be such that $\sigma(\alpha)=$ $d(\sigma) \cdot \alpha$. As $\sigma(\alpha) \in Z$, we have by Lemma 3.10 that $d(\sigma) \in\left(G, s_{1}\right)^{\sharp}(L)$. As any $\beta \in Z$ is of the form $\alpha \cdot c$ for $c \in(G, s)^{\sharp}(L)=(G, s)^{\sharp}(K)$ and $\sigma$ fixes $K$ pointwise, we see that

$$
\sigma(\beta)=d(\sigma) \cdot \beta \text { for all } \beta \in Z .
$$

We only have to see that $d(\sigma) \in S$. Let $X$ be any $D$-subvariety of $\left(G, s^{\prime}\right)$ defined over $K$. As $\hat{K}$ is differentially closed and $Z=\left(G, s^{\prime}\right)^{\sharp}(\hat{K})$, it follows that $Z \cap X$ is Zariski-dense in $X$. For $\beta \in Z \cap X, \sigma(\beta) \in Z \cap X$ too. By $(*)$ and Zariski-denseness, $d(\sigma) \cdot X=X$. Thus $d(\sigma) \in S$.

Conversely, suppose that $g \in\left(S, s_{1}\right)^{\sharp}(L)$. Then $g \cdot \alpha \in Z$. Let $X$ be the algebraic variety defined over $K$ whose $K$-generic point is $\alpha$. Then, $X$ is a $D$-subvariety of $\left(G, s^{\prime}\right)$. Thus $g \cdot X=X$ and so $g \cdot \alpha \in X$, and $\partial(g \cdot \alpha)=s^{\prime}(g \cdot \alpha)$. Hence $g \cdot \alpha$ is a differential specialization of $\alpha$ over $K$. By simplicity of $K[\alpha], \alpha$ and $g \cdot \alpha$ satisfy exactly the same differential polynomial equations over $K$. As both $\alpha$ and $g \cdot \alpha$ generate $L$ it follows that there is an automorphism $\sigma$ of $L$ over $K$ such that $\sigma(\alpha)=g \cdot \alpha$. So $g=d(\sigma)$.

Conclusion of proof of Lemma 3.9. Let $H$ be the image of $S$ under conjugation by $\alpha^{-1}$ in $G\left(g \rightarrow \alpha^{-1} \cdot g \cdot \alpha\right)$. Then $H$ is an algebraic $D$-subgroup of $(G, s)$. By 3.11 and what we already know about 3.9 , the image of the embedding $c$ : $\operatorname{Aut}_{\partial}(L / K) \rightarrow(G, s)^{\sharp}(K)$ is precisely $(H, s)^{\sharp}(K)$. As the latter is Zariski-dense in $H, H$ is also defined over $K$.

Let us fix the isomorphism $c$ between $\operatorname{Aut}_{\partial}(L / K)$ and $(H, s)^{\sharp}(K)=$ $(H, s)^{\sharp}(\hat{K})$.

Lemma 3.12. There is a Galois correspondence between the set of differential fields in between $K$ and $L$ and the set of algebraic $D$-subgroups of 
$(H, s)$ defined over $K$ (equivalently defined over $\hat{K})$ : given $K<F<L$ the corresponding group is $H_{F}$ the Zariski closure of the set of $h \in(H, s)^{\sharp}(K)$ such that $c^{-1}(h)$ is the identity on $F$.

Proof. This is Theorem 2.12 of [8] after making the translation between definable subgroups and $D$-subgroups.

Let us complete this section with an example. We will consider the nonisotrivial algebraic $D$-group structure $(G, s)$ on $G_{m} \times G_{a}$ discussed at the end of Section 2, and exhibit a (natural) differential Galois extension $K<L$ whose differential Galois group is $(G, s)$ (or rather $(G, s)^{\sharp}(K)$ ). In fact there will be an intermediary differential field $K<F<L$ such that each of $K<F$ and $F<K$ are Picard-Vessiot extensions, but $K<L$ is not a Picard-Vessiot extension.

Recall that $s: G \rightarrow T(G)$ is given by $s(x, y)=(x, y, x y, 0)$, and $l D_{s}:$ $G \rightarrow L(G)$ is $l D_{s}(x, y)=(\partial(x) / x-y, \partial(y))$, and so a logarithmic differential equation on $(G, s)$ has the form $\left\{\partial(x) / x-y=a_{1}, \partial(y)=a_{2}\right\}$. If we restrict our attention to equations where $a_{1}=0$, we obtain equations of the form $\partial(\partial(x) / x)=a$ on $G_{m}$.

Also $(G, s)^{\sharp}$ can be identified with $\left.\left\{x \in G_{m}: \partial(\partial(x) / x)\right)=0\right\}$.

Note that the embedding $x \rightarrow(x, 0)$ of $G_{m}$ in $G$ and surjection $(x, y) \rightarrow y$ of $G$ onto $G_{a}$ induces an exact sequence

$$
0 \rightarrow\left(G_{m},\left(s_{0}\right)_{G_{m}}\right) \rightarrow(G, s) \rightarrow\left(G_{a},\left(s_{0}\right)_{G_{a}}\right) \rightarrow 0
$$

of algebraic $D$-groups, where $s_{0}$ denotes the 0 -sections for the corresponding groups. The important fact is that $(G, s)$ is not a product (as a $D$-group) of the two groups, which is the reason that $(G, s)$ is nonisotrivial.

We will take the ground field of constants to be $\mathbf{C}$. Let $K=\mathbf{C}\left(e^{c t}: c \in \mathbf{C}\right)$ and $L=K\left(t, e^{t^{2}}\right)$. So $L$ is a subfield of a fixed differential closure $\hat{\mathbf{C}}=\hat{K}=\hat{L}$ of $\mathbf{C}$.

\section{Lemma 3.13. $(G, s)$ is $K$-large.}

Proof. It is enough to show that all solutions of $\partial(\partial(x) / x)=0$ in $\hat{K}$ are in $K$, that is all solutions of $\partial(d) / d=c$ for $c \in \mathbf{C}$ that are in $\hat{K}$ are in $K$. But this is clear, because $e^{c t}$ is one such such solution and the others are obtained by multiplying by a constant.

Lemma 3.14. $L$ is a differential Galois extension of $K$ for the equation $\partial(\partial(x))=2$. The Galois group is $(G, s)^{\sharp}(K)$.

Proof. Note that $L$ is generated over $K$ as a differential field by $e^{t^{2}}$, which is a solution of $\partial(\partial(x))=2$. To show that the Galois group is as stated, it is enough to show that $\operatorname{tr} \cdot \operatorname{deg}(L / K)=2$, which is well-known.

Note that $K(t)$ is a Picard-Vessiot extension of $K$, and $L$ is a PicardVessiot extension of $K(t)$, but $L$ is not a Picard-Vessiot extension of $K$. 


\section{Isomorphisms of algebraic $D$-groups and algebraic $D$-varieties}

We will prove:

Proposition 4.1. Suppose $(K, \partial)$ is an algebraically closed differential field, $(G, s)$ and $(H, t)$ are connected algebraic D-groups over $K$ and there is an isomorphism $f$ between $(G, s)$ and $(H, t)$ defined over some differential field extension of $K$. Then there is such an isomorphism defined over a PicardVessiot extension of $K$.

Here is a restatement of the theorem in the language of Kolchin's constrained cohomology (see [5]).

Corollary 4.2. Suppose $(K, \partial)$ has no proper Picard-Vessiot extensions. Then for any connected $\partial_{0}$-group $G$ defined over $K, H_{c}^{1}\left(\right.$ Aut $\left._{\partial}(\hat{K} / K), G(\hat{K})\right)$ is trivial.

In Proposition 3.11 of [9], Corollary 4.2 was stated in the special case that $H(K)=H(\hat{K})$ (which amounts to saying that the corresponding algebraic $D$-group is $K$-large).

Kolchin's differential tangent space and its properties (see Chapter 8 of [5]) play an important role in the proof of Proposition 4.1. We will summarize the key properties (in the language of algebraic $D$-groups). Recall first that if $V$ is a finite-dimensional vector space over $\mathcal{U}$, then a $\partial$-module structure on $V$ is an additive map $D_{V}: V \rightarrow V$ such that $D_{V}(a v)=$ $\partial(a) v+a D_{V}(v)$ for all $a \in \mathcal{U}$ and $v \in V$. $V^{\partial}$ denotes $\left\{v \in V: D_{V}(v)=0\right\}$, a vector space over $\mathcal{C}$ with $\mathcal{C}$-dimension the same as the $\mathcal{U}$-dimension of $V$

Fact 4.3. Suppose $(G, s)$ is a connected algebraic $D$-group defined over $K$, and $V=L(G)$ is its Lie algebra (tangent space at the identity). Then:

(i) $s$ equips $V$ with a canonical $\partial$-module structure $D_{V}$, defined over $K$.

(ii) For any automorphism $f$ of $(G, s), d f_{e} \in \mathrm{GL}(V)$ restricts to a $\mathcal{C}$-linear automorphism of $V^{\partial}$, and moreover $f$ is determined by $d f_{e} \mid V^{\partial}$.

Proof of Proposition 4.1. First $(G, s)$ and $(H, t)$ will be isomorphic over $\hat{K}$. Let $f$ be such an isomorphism. Let $c$ be a finite tuple from $\hat{K}$ generating the smallest field of definition of $f$. Write $f$ as $f_{c}$. Let $\left(V, D_{V}\right)$ be as in Fact 4.3 for $(G, s)$. Let $d$ be a $\mathcal{C}$-basis for $V^{\partial}$ contained in $\hat{K}$. Let $L=K\langle c, d\rangle$ be the differential field generated by $c$ and $d$ over $K$.

Claim I. $L$ is a strongly normal extension of $K$.

Proof. As $L<\hat{K}, C_{L}=C_{K}$. We have to show that for any automorphism $\sigma$ of the differential field $\mathcal{U}$ fixing $K$ pointwise, $\sigma(L)$ is contained in the differential field generated by $L$ and $\mathcal{C}$. First $\sigma(d)$ is another basis of $V^{\partial}$, so with respect to the basis $d$ we may write $\sigma(d)$ as a $n \times n$ nonsingular matrix $B \in \mathrm{GL}_{n}(\mathcal{C})$. On the other hand $f_{\sigma}(c)=f_{c} \cdot h$ for a unique automorphism 
$h$ of $(G, s)$. By Fact 4.3 (ii), $h$ is determined by $d h_{e} \mid V^{\partial}$, which by in terms of the basis $d$, is another nonsingular $n \times n$ matrix $A$ over $\mathcal{C}$. It follows that $(\sigma(c), \sigma(d))$ is in the differential field generated by $K, c, d, A$ and $B$. In particular $\sigma(L) \subseteq L\langle\mathcal{C}\rangle$.

Claim II. $L$ is a Picard-Vessiot extension of $K$.

Proof. Let $\sigma \in \operatorname{Aut}_{\partial}(L / K)$, and write the matrices $A, B$ (which will be in $\left.\mathrm{GL}_{n}\left(C_{K}\right)\right)$ as $A_{\sigma}, B_{\sigma}$. Then it is easy to check that $\sigma \mapsto\left(A_{\sigma}, B_{\sigma}\right)$ is an embedding of Aut ${ }_{\partial}(L / K)$ into $\mathrm{GL}_{n}\left(C_{K}\right) \times \mathrm{GL}_{n}\left(C_{K}\right)$. From Kolchin's characterizations of Picard-Vessiot extensions, we get Claim II.

As $f$ is defined over $L$, we have proved Proposition 4.1.

Finally let us give some additional results with a similar flavor. The first is really just a remark and is related to the themes of Buium's book [2]. Buium calls an algebraic $D$-variety $(X, s)$ split if $(X, s)$ is isomorphic (over $\mathcal{U})$ to a trivial algebraic $D$-variety, namely one of the form $\left(Y, s_{0}\right)$, where $Y$ is defined over $\mathcal{C}$ and $s_{0}$ is the 0 -section. He proves that any $D$-variety $(X, s)$ such that $X$ is a projective variety is split. Moreover assuming $Y$ defined over algebraically closed $K$, then $(Y, s)$ is split over some strongly normal extension.

Remark 4.4. Let $K$ be a differential field with algebraically closed constant field. Suppose that $(X, s)$ is an algebraic D-variety over $K$ that is split. Then $(X, s)$ is split over some strongly normal extension $K_{1}$ of $K$.

Proof. We can find an isomorphism $f$ of $(X, s)$ with some trivial $\left(Y, s_{0}\right)$ defined over $\hat{K}$. Let again $f=f_{c}$ with $c$ the smallest field of definition of $f$. Let $K_{1}=K\langle c\rangle$. Then, as $K_{1}$ is contained in $\hat{K}$ and $C_{\hat{K}}=C_{K}$, we have that $C_{K_{1}}=C_{K}$. For any (differential) automorphism $\sigma$ of $\mathcal{U}$, Then $f_{\sigma(c)}=f_{c} \circ g$ for some automorphism $g$ of $\left(Y, s_{0}\right)$. But then $g$ must be defined over $\mathcal{C}$. Hence $\sigma(c)$ is rational over $K(c)(\mathcal{C})$, so $\sigma\left(K_{1}\right) \subseteq K_{1}\langle\mathcal{C}\rangle$. This shows that $K_{1}$ is a strongly normal extension of $K$.

The next result is a generalization of Proposition 4.1 in which the higherdimensional versions of differential tangent spaces from [10] enter the picture. We use freely the results from that paper.

Proposition 4.5. Suppose that $K$ has algebraically closed constant field. Let $(X, s)$ and $(Y, t)$ be algebraic D-varieties defined over $K$. Suppose that $a \in(X, s)^{\sharp}(K), b \in(Y, t)^{\sharp}(K)$ are nonsingular points on $X, Y$ respectively, and that there is some isomorphism $f$ between $(X, s)$ and $(Y, t)$ such that $f(a)=b$. Then there is such an isomorphism defined over a Picard-Vessiot extension of $K$. 
Proof. For each $m \geq 1$, let $V_{m}$ the the $\mathcal{U}$-vector space $\mathcal{M} / \mathcal{M}^{m+1}$, where $\mathcal{M}$ is the maximal ideal of the local ring of $X$ at $a . V_{m}$ is defined over $K$. For any automorphism $h$ of $X$ such that $h(a)=a, h$ induces a linear automorphism $h_{m}$ say of $V_{m}$. Moreover if $h^{\prime}$ is another automorphism of $X$ fixing $a$, then $h=h^{\prime}$ if and only if $h_{m}=h_{m}^{\prime}$ for all $m$. So far nothing has been said about the $D$-variety structure. As $a \in(X, s)^{\sharp}, \partial$ extends to a derivation $\partial^{\prime}$ on the local ring of $X$ at $a$ that preserves $\mathcal{M}$ and all its powers. This gives $V_{m}$ the structure of a $\partial$ module $\left(V_{m}, D_{V_{m}}\right)$ over $\mathcal{U}$, defined over $K$ (for all $m$ ). If $h$ is an automorphism of the algebraic $D$-variety $(X, s)$ that fixes $a$ then $h_{m} \in \mathrm{GL}\left(V_{m}\right)$ restricts to a $\mathcal{C}$-linear automorphism $h_{m}^{\partial}$ of $\operatorname{GL}\left(V_{m}^{\partial}\right)$ (where $V_{m}^{\partial}$ is the solution space of $D_{V_{m}}=0$ ). Moreover $h_{m}$ is determined by $h_{m}^{\partial}$.

Now we may find an isomorphism $f$ between $(X, s)$ and $(Y, t)$ such that $f(a)=b$ and $f$ is defined over $\hat{K}$. Let $c$ generate the smallest field of definition of $f$. So $c$ is a finite tuple from $\hat{K}$. Write $f$ as $f_{c}$. For any differential automorphism $\sigma$ of $\mathcal{U}$ that fixes $K$ pointwise, $\sigma\left(f_{c}\right)=f_{\sigma(c)}$ is also an isomorphism of $(X, s)$ with $(Y, t)$ taking $a$ to $b$. Hence $f_{\sigma(c)} \circ f_{c}^{-1}$ is an automorphism of $(X, s)$ taking $a$ to itself. Write $h^{\sigma}$ for this map.

Claim I. There is $m$ such that for all $\sigma, \tau \in$ Aut $_{\partial}(\mathcal{U} / K), h^{\sigma}=h^{\tau}$ if and only if $\left(h_{m}^{\sigma}\right)^{\partial}=\left(h_{m}^{\tau}\right)^{\partial}$.

Proof. This follows from compactness and the earlier remarks as the set of $h^{\sigma}$ is a uniformly definable family of automorphisms of $(X, s)$.

Now let $m$ be as in Claim I and let $d$ be a $\mathcal{C}$-basis for $\left(V_{m}\right)^{\partial}$.

Claim II. $K_{1}=K\langle c, d\rangle$ is a strongly normal extension of $K$.

Proof. Let $\sigma \in \operatorname{Aut}_{\partial}(\mathcal{U} / K)$. As $\sigma(d)$ is also a basis for $\left(V_{m}\right)^{\partial}, \sigma(d) \in$ $K\langle d\rangle\langle\mathcal{C}\rangle$. On the other hand, by virtue of $d,\left(V_{m}\right)^{\partial}$ can be identified with $\mathcal{C}^{r}$ for suitable $r$, and thus $\left(h_{m}^{\sigma}\right)^{\partial}$ can be identified with an element of $\mathrm{GL}_{r}(\mathcal{C})$. By Claim I, it follows that $\sigma(c) \in K\langle c, d\rangle\langle\mathcal{C}\rangle$. Thus $\sigma\left(K_{1}\right) \subseteq K_{1}\langle\mathcal{C}\rangle$.

As in the proof of 4.1, we conclude that actually $K_{1}$ is a Picard-Vessiot extension of $K$. As $c \in K_{1}$, this gives the proposition.

Acknowledgements. This work was carried out while the author was an Invited Professor at the University of Paris VI in June 2003, and while visiting the University of Naples II in July 2003. I am indebted to Daniel Bertrand at Paris VI for his hospitality, many helpful conversations, and his interest in the work reported here. Thanks also to Zoe Chatzidakis of University Paris VII-CNRS for her suggestions, and to Paola D'Aquino of Naples II for her hospitality. 


\section{References}

[1] D. Bertrand, Review of "Lectures on differential Galois theory", by A. Magid, Bull. Amer. Math. Soc., 33 (1996), 289-294.

[2] A. Buium, Differential Function Fields and Moduli of Algebraic Varieties, Lecture Notes in Mathematics, 1226, Springer, 1986, MR 0874111 (88e:14010), Zbl 0613.12018 .

[3] A. Buium, Differential Algebraic Groups of Finite Dimension, Lecture Notes in Mathematics, 1506, Springer, 1992, MR 1176753 (93i:12010), Zbl 0756.14028.

[4] E. Kolchin, Differential Algebra and Algebraic Groups, Pure and Applied Mathematics, 54, Academic Press, New York, 1973, MR 0568864 (58 \#27929), Zbl 0264.12102.

[5] E. Kolchin, Differential Algebraic Groups, Pure and Applied Mathematics, 114, Academic Press, Orlando, 1985, MR 0776230 (87i:12016), Zbl 0556.12006.

[6] D. Marker, Manin kernels, in 'Connections between Model Theory and Algebraic and Analytic Geometry', Quaderni di Matematica, Univ. Naples II, 2000, MR 1930680 (2003g:12009).

[7] D. Pierce and A. Pillay, A note on the axioms for differentially closed fields of characteristic zero, J. Algebra, 204 (1998), 108-115, MR 1623945 (99g:12006), Zbl 0922.12006.

[8] A. Pillay, Differential Galois theory I, Illinois J. Math., 42 (1998), 678-699, MR 1649893 (99m:12009), Zbl 0916.03028.

[9] A. Pillay, Finite-dimensional differential algebraic groups and the Picard-Vessiot theory, in 'Differential Galois Theory', Banach Center Publications, 58, Polish Academy of Sciences, 2002, 189-199, MR 1972454 (2004c:12008), Zbl 1036.12006.

[10] A. Pillay and M. Ziegler, Jet spaces of varieties over differential and difference fields, Selecta Math., 9(4) (2003), 579-599, MR 2031753.

[11] B. Poizat, A Course in Model Theory. An Introduction to Contemporary Mathematical Logic, Universitext, Springer, New York, 2000, MR 1757487 (2001a:03072), Zbl 0951.03002.

[12] M. van der Put and M. Singer, Galois Theory of Linear Differential Equations, Grundlehren der Mathematischen Wissenschaften, 328, Springer, Berlin, 2003, MR 1960772 (2004c:12010), Zbl 1036.12008.

Received January 20, 2004 and revised May 7, 2004. The author was supported by NSF grant DMS 0300639 and NSF Focused Research Grant DMS 0100979.

Department of Mathematics

UNIVERSity OF ILLINOIS AT URBANA-CHAMPAIGN

URBANA IL 61801-2975

E-mail address: pillay@math.uiuc.edu 